\title{
Targeting the Mitotic Checkpoint to Kill Tumor Cells
}

\author{
Aniek Janssen • Geert J. Kops • René H. Medema
}

Published online: 17 December 2010

(C) The Author(s) 2010. This article is published with open access at Springerlink.com

\begin{abstract}
One of the most common hallmarks of cancer cells is aneuploidy or an abnormal number of chromosomes. This abnormal chromosome content is a consequence of chromosome missegregation during mitosis, a defect that is seen more frequently in tumor cell divisions as in normal cell divisions. In fact, a large fraction of human tumors display a chromosome instable phenotype, meaning that they very frequently missegregate chromosomes. This can cause variegated aneuploidy within the tumor tissue. It has been argued that this hallmark of cancer could be exploited in anti-cancer therapies. Here we test this hypothesis by inactivation of the mitotic checkpoint through RNAi-mediated depletion of an essential checkpoint component, Mps1. The mitotic checkpoint delays segregation of chromosomes during mitosis until all chromosomes are properly attached to the mitotic spindle. Its inactivation will therefore lead to increased segregation errors. Indeed, we show that this can lead to increased cell death in tumor cells. We demonstrate that increased cell death is associated with a dramatic increase in segregation errors. This suggests that inhibition of the mitotic checkpoint might represent a useful anti-cancer strategy.
\end{abstract}

Keywords Aneuploidy · Chromosomal instability · Mps1

\footnotetext{
A. Janssen $\cdot$ R. H. Medema $(\bowtie)$

Department of Medical Oncology and Cancer Genomics Center, University Medical Center Utrecht, Universiteitsweg 100,

3584 CG, Utrecht, The Netherlands

e-mail: r.h.medema@umcutrecht.nl

G. J. Kops

Department of Physiological Chemistry,

University Medical Center Utrecht,

Universiteitsweg 100 ,

3584 CG, Utrecht, The Netherlands
}

\section{Introduction}

Chromosomal instability (CIN) is a common characteristic of solid tumors that is manifested as gains or losses of whole chromosomes during cell division, leading to aneuploidy [1]. This propensity of tumor cells was recognized more than 100 years ago [2], leading to the hypothesis that $\mathrm{CIN}$ could be a driving force in tumorigenesis [3]. Indeed, several lines of evidence have since suggested a causal role for CIN in tumor formation (reviewed in [4]). However, the exact cause of CIN in tumors has remained largely unknown.

It has become clear that a broad range of mutations in different cellular processes can result in CIN [5]. Defects in mitotic checkpoint function have also been invoked as a causal event for CIN [5]. The mitotic checkpoint ensures the fidelity of sister chromatid segregation over the two daughter cells by inhibiting progression to anaphase until all chromosome pairs are bi-oriented on the mitotic spindle (reviewed in [6]). In various human cancers, mitotic checkpoint function is partially compromised, and altered expression or mutations of mitotic checkpoint genes have been shown to be related to CIN and aneuploidy (reviewed in $[5,7])$. Nevertheless, no evidence for checkpoint malfunctions as a direct cause of CIN in tumor cells has been found [8-10]. Moreover, the mitotic checkpoint defects that have been reported (reviewed in [5]) may be well tolerated by the tumor cell population and most likely do not result in complete checkpoint inactivation. Complete inactivation of the mitotic checkpoint, however, results in gross chromosomal missegregation and is not compatible with cell viability [11-14]. This has led to the suggestion that inhibition of the mitotic checkpoint could have therapeutic potential in cancer treatment [7]. Moreover, tumor cells that have acquired a decreased checkpoint activity could be more sensitive to mitotic checkpoint 
inhibition when compared to healthy, checkpoint proficient, cells (reviewed in [7]).

Here, we have explored the relationship between chromosome missegregations and tumor cell death by using conditional depletion of the mitotic checkpoint component Mps1.

\section{Materials and Methods}

Tissue Culture, Transfections, and Treatments

LS174T cells were grown in DMEM (Lonza) with $10 \%$ Tetapproved FCS (Clontech), supplemented with pen/strep (Invitrogen) and ultraglutamine (Lonza). Taxol, MG132, and doxycycline (used at $1 \mu \mathrm{g} / \mathrm{ml}$ ) were from Sigma. LS174T cells expressing TetR were infected with retrovirus carrying pSuperior-retro-puro-Mps1 and selected with $2 \mu \mathrm{g} / \mathrm{ml}$ of puromycin. Single colonies were selected after replating 1-2 cells/well.

\section{Immunofluorescence Microscopy}

Cells plated on 12-mm coverslips were harvested after 90min MG132 treatment. Fixation was done using 4\% PFA in PEM buffer. CREST was incubated O/N in PBS 3\% BSA. Anti-human Alexafluor647 and DAPI were incubated in PBS $0.1 \%$ Tween. Stained coverslips were mounted with Vectashield Mounting Medium (Vector). Images were acquired on a Zeiss 510 Meta confocal laser scanning microscope with a $63 \times / 1$.4NA Plan-ApoChromat objective using the Zeiss LSM software.

\section{Chromosome Spreads}

Nocodazole was added for $4 \mathrm{~h}$ to the medium to enrich for mitotic cells. Cells were treated with $0.075 \mathrm{M} \mathrm{KCl}$ at $37^{\circ} \mathrm{C}$ for $10 \mathrm{~min}$, centrifuged at 2,000 rpm, and fixed for $20 \mathrm{~min}$ with methanol/acetic acid (3:1). Fixation procedure was repeated three times. Samples were collected in methanol and DAPI to stain for DNA. Chromosome spreads were created by allowing the drops to fall from $30 \mathrm{~cm}$ height onto glass slides. Images were acquired as described above for immunofluorescence.

\section{Flow Cytometry}

Flow cytometry samples were harvested and fixed using $70 \%$ ethanol. $\alpha$-MPM2 was incubated for $1 \mathrm{~h}$ in PBS-2\% BSA- $-0.1 \%$ Tween and $\alpha$-Mouse Cy 5 for $1 \mathrm{~h}$ in PBS- $-0.1 \%$ Tween. Stained cells were collected in PBS containing RNAse and propidium iodide. Fluorescence was measured on the FACSCalibur and analyzed with Cell Quest Pro software (BD Biosciences).
Immunoblotting and Antibodies

Cells were lysed in Laemmli buffer. Samples were separated by SDS-PAGE and transferred to PVDF (Immobilin FL; Millipore). The membranes were cut in half and blotted with anti-Mps1 and anti- $\alpha$-tubulin. The following antibodies have been used for western blot, immunofluorescence, and FACS analysis: Mps1 (Upstate), $\alpha$-tubulin (Sigma), CREST (Cortex Biochem), MPM2 (Upstate), antihuman Alexafluor647, and anti-mouse cy-5 (Jackson)

\section{Colony Formation Assays}

Cells $( \pm 50,000 /$ well $)$ were plated on six-well plates (Costar). Doxycycline was added at day 0 to allow knockdown of the proteins. At day 11, plates were washed with PBS, fixed 5 min with $96 \%$ methanol, and stained with $0.1 \%$ crystal violet.

\section{Results and Discussion}

Efficient Mps1 Depletion Severely Compromises Viability of Tumor Cells

We have previously shown that absence of Mad2, BubR1, or Mps1 causes cell death within six cell divisions [14-17]. To examine the effect of conditional inactivation of Mps1 in a mitotic checkpoint proficient cell line, we stably introduced a doxycycline-inducible vector encoding a small hairpin RNA (shRNA) directed against human Mps1 in LS174-T colon carcinoma cells. In addition, this line stably expresses a tet-repressor such that the addition of tetracycline or doxycycline to the culture medium induces the expression of the Mps1 shRNA and a consequent depletion of the endogenous Mps1 protein [15]. A clone was selected in which expression of Mps1 was reduced by more than $90 \%$ upon addition of doxycycline to the culture medium (Fig. 1a). This degree of depletion of Mps1 severely compromised the viability of the LS174-T cells since no colonies grew out in the presence of doxycycline (Fig. 1a). Thus, selective inhibition of Mps1 compromises the viability of LS174-T tumor cells.

Mps1 Depletion Inactivates the Spindle Checkpoint

Mps1 is an essential component of the spindle checkpoint, and its inactivation has been shown to override the cellular response to spindle poisons such as nocodazole and taxol $[18,19]$. Therefore, we next tested if RNAi-mediated depletion of Mps1 in the LS174-T cells conditionally expressing the Mps1 shRNA leads to functional inactivation of Mps1. To this end, we cultured these cells in the 


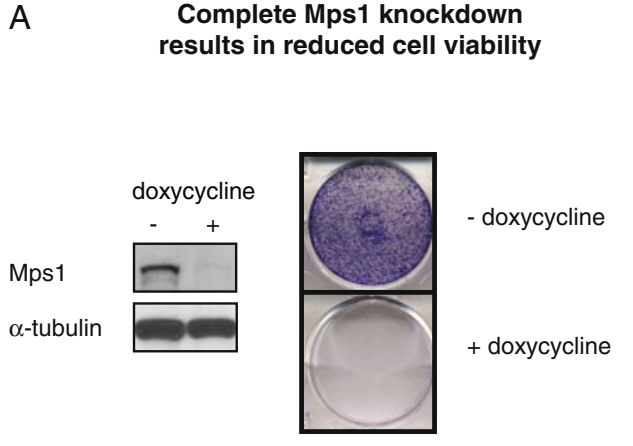

Fig. 1 Conditional inactivation of Mps1 causes inactivation of the mitotic checkpoint and tumor cell death. a Left LS174T-TetRMps1, treated without $(-)$ and with $(+)$ doxycycline (dox) for 3 days, were immunoblotted for Mps1 and $\alpha$-tubulin. Right colony formations of LS174T-TetRMps 1 cells treated with and without dox for 11 days. b

presence or absence of doxycycline for a period of 3 days and added $1 \mu \mathrm{M}$ taxol to the culture medium for the last $18 \mathrm{~h}$ of the incubation period. Taxol stabilizes microtubules and perturbs proper spindle assembly. This will normally lead to a mitotic delay and an accumulation of cells in mitosis that is dependent on the mitotic checkpoint. Indeed, in LS174-T cells that are grown in the absence of doxycycline, we find a clear accumulation of cells in mitosis of up to $75 \%$ of all cells (Fig. 1b). In contrast, depletion of Mps1 by the addition of doxycycline leads to clear inactivation of the mitotic checkpoint since less than $5 \%$ of the cells are mitotic in these cultures (Fig. 1b). Addition of doxycycline to the parental LS174 cells did not result in a checkpoint override, indicating that this effect was due to specific depletion of Mps1 (data not shown).
B

Mitotic Checkpoint deficiency

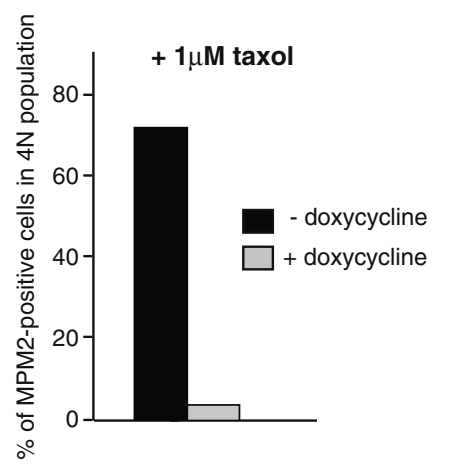

FACS analysis of LS174T-TetRMps1 cells treated with or without dox for 3 days and $1 \mu \mathrm{M}$ taxol for $18 \mathrm{~h}$. The percentage of mitotic cells was measured as the fraction of cells with 4 N DNA content that were positive for MPM2

These results indicate that conditional depletion of Mps1 from LS174-T tumor cells leads to inactivation of the mitotic checkpoint and a concomitant reduction in cell viability.

Mps1 Depletion Causes Severe Chromosome Alignment Defects and Enhanced Aneuploidy

Inactivation of Mps1 is expected to compromise a cell's ability to achieve proper chromosome alignment on the mitotic spindle [17]. Therefore, we analyzed if conditional depletion of Mps1 compromised the fidelity of chromosome alignment in LS174-T tumor cells. To this end, LS174-T cells grown in the presence or absence of doxycycline for 3 days were incubated with the proteasome

\section{A}

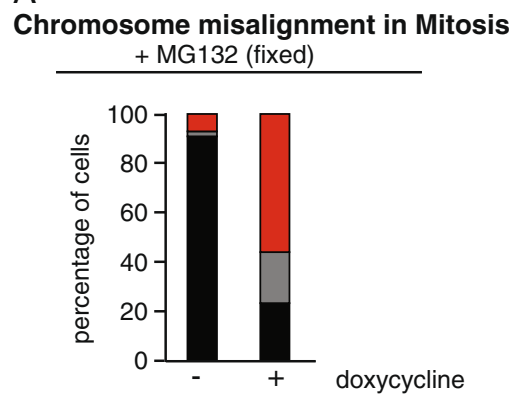

no alignment defects

$\square$ mild alignment defects (1-5 chromosomes)

$\square$ severe alignment defects (>5 chromosomes)

Fig. 2 Conditional inactivation of Mps1 causes severe defects in chromosome segregation and severe aneuploidy. a LS174T-TetRMps1 cells were treated with or without dox for 3 days and fixed after 90 min of MG132 treatment. No alignment defects, mild alignment defects, or severe alignment defects indicate mitotic cells with zero,

B

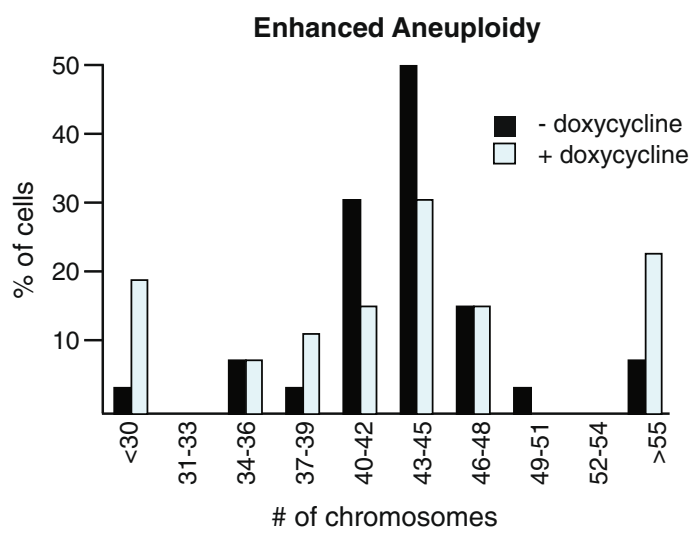

one to five, or more than five chromosomes not aligned on the metaphase plate, respectively. b Chromosome spreads were performed on cells after 4 days of treatment with or without dox. At least 60 chromosome spreads were counted per condition 
inhibitor MG132 for 90 min to arrest cells at the metaphaseto-anaphase transition. In control cells grown in the absence of doxycycline, we found that $90 \%$ of all mitotic cells had fully aligned their chromosomes (Fig. 2a). In contrast, depletion of Mps1 by the addition of doxycycline to the culture medium resulted in a severe reduction, as less than $25 \%$ of the cells managed to align their chromosomes in this time (Fig. 2a). This increase in segregation errors coincides with a more severe aneuploidy, as evidenced by the karyotyping shown in Fig. 2b. Taken together, these data show that conditional inactivation of Mps1 compromises the mitotic checkpoint and results in a severe defect in chromosome alignment. The combination of these two effects results in enhanced segregation errors and as a consequence the karyotype of the tumor cells becomes highly unstable.

Taken together, our data demonstrate that conditional inactivation of Mps1 inactivates the mitotic checkpoint, increases chromosome alignment defects, and compromises cell viability in vitro. Our data show that inactivation of Mps1 can inhibit tumor growth and suggest that targeting Mps1 function might be a useful anti-cancer strategy. Future studies will have to resolve if partial inhibition of Mps1 can perturb chromosome segregation in vivo and whether this is a useful strategy to interfere with tumor growth in vivo. For this purpose, we would like to develop pharmacological inhibitors of Mps1 and intend to perform intravital imaging of cell division of xenografted tumor cells in the mice. These studies will show if inhibition of Mps1 induces gross chromosome missegregation in vivo and whether this is associated with tumor-selective cell death.

Open Access This article is distributed under the terms of the Creative Commons Attribution Noncommercial License which permits any noncommercial use, distribution, and reproduction in any medium, provided the original author(s) and source are credited.

\section{References}

1. Lengauer C, Kinzler KW, Vogelstein B (1998) Genetic instabilities in human cancers. Nature 396(6712):643
2. von Hansemann DP (1890) Über asymmetrische Zelltheilung in Epithelkrebsen und deren biologische Bedeutung. Arch Pathol Anat Physiol Klin Med 779:299

3. Boveri Theodor (1914) Zur Frage der Entstehung maligner Tumoren vol. 1

4. Suijkerbuijk SJ, Kops GJ (2008) Preventing aneuploidy: the contribution of mitotic checkpoint proteins. Biochim Biophys Acta 1786:24-31

5. Weaver BA, Cleveland DW (2006) Does aneuploidy cause cancer? Curr Opin Cell Biol 18(6):658

6. Musacchio A, Salmon ED (2007) The spindle-assembly checkpoint in space and time. Nat Rev Mol Cell Biol 8(5):379

7. Kops GJ, Weaver BA, Cleveland DW (2005) On the road to cancer: aneuploidy and the mitotic checkpoint. Nat Rev Cancer 5(10):773

8. Gascoigne KE, Taylor SS (2008) Cancer cells display profound intra- and interline variation following prolonged exposure to antimitotic drugs. Cancer Cell 14:111-122

9. Thompson SL, Compton DA (2008) Examining the link between chromosomal instability and aneuploidy in human cells. J Cell Biol 180(4):665

10. Tighe A, Johnson VL, Albertella M, Taylor SS (2001) Aneuploid colon cancer cells have a robust spindle checkpoint. EMBO Rep 2 (7):609

11. Dobles $M$ et al (2000) Chromosome missegregation and apoptosis in mice lacking the mitotic checkpoint protein Mad2. Cell 101 (6):635

12. Kalitsis P, Earle E, Fowler KJ, Choo KH (2000) Bub3 gene disruption in mice reveals essential mitotic spindle checkpoint function during early embryogenesis. Genes Dev 14(18):2277

13. Michel L et al (2004) Complete loss of the tumor suppressor MAD2 causes premature cyclin B degradation and mitotic failure in human somatic cells. Proc Natl Acad Sci USA 101 (13):4459

14. Kops GJ, Foltz DR, Cleveland DW (2004) Lethality to human cancer cells through massive chromosome loss by inhibition of the mitotic checkpoint. Proc Natl Acad Sci USA 101 (23):8699

15. Janssen A, Kops GJPL, Medema RH (2009) Elevating the frequency of chromosome missegregation as a strategy to kill tumor cells. PNAS 106:19108-19113

16. Jelluma $\mathrm{N}$ et al (2008) Chromosomal instability by inefficient Mps1 auto-activation due to a weakened mitotic checkpoint and lagging chromosomes. PLoS ONE 3(6):e2415

17. Jelluma $\mathrm{N}$ et al (2008) Mps1 phosphorylates Borealin to control Aurora B activity and chromosome alignment. Cell 132(2):233

18. Abrieu A et al (2001) Mps1 is a kinetochore-associated kinase essential for the vertebrate mitotic checkpoint. Cell 106(1):83

19. Stucke VM, Sillje HH, Arnaud L, Nigg EA (2002) Human Mps1 kinase is required for the spindle assembly checkpoint but not for centrosome duplication. EMBO J 21(7):1723 